\title{
Detection and analysis of the effects of heat stress on EEG using wavelet transform -EEG analysis under heat stress
}

\author{
Prabhat Kumar Upadhyay ${ }^{1}$, Rakesh Kumar Sinha ${ }^{2}$, Bhuwan Mohan Karan ${ }^{1}$ \\ ${ }^{1}$ Department of Electrical and Electronics Engineering Birla Institute of Technology, Birla, India; \\ ${ }^{2}$ Department of Biomedical Instrumentation Birla Institute of Technology, Birla, India. \\ Email: uprabhat@rediffmail.com
}

Received 25 October 2009; revised 17 November 2009; accepted 6 December 2009.

\begin{abstract}
Continuous wavelet transform (CWT) method has been applied to capture localized time-frequency information of rat electroencephalogram (EEG) in different vigilance states and analyze alterations in transients during awake, slow wave sleep (SWS), and rapid eye movement (REM) sleep stages due to exposure to high environmental heat. Rats were divided in three group (i) acute heat stress-subjected to a single exposure for four hours in the Biological Oxygen Demand (BOD) incubator at $38^{\circ} \mathrm{C}$; (ii) chronic heat stress-exposed for 21 days daily for one hour in the incubator at $38^{\circ} \mathrm{C}$, and (iii) handling control groups. After two hours long EEG recordings from young healthy rats, EEG data representing three sleep states was visually selected and further subdivided into 2 seconds long epoch. Powers of wavelet spectra corresponding to delta, theta, alpha, and beta bands at all scales and locations were computed and variation in their states investigated. The wavelet analysis of EEG signals following exposure to high environmental heat revealed that powers of subband frequencies vary with time unlike Fourier technique. Changes in higher frequency components (beta) were significant in all sleep-wake states following both acute and chronic heat stress conditions. Percentage power of different components of the four bands was always found to be varying at different intervals of time in the same signal of analysis.
\end{abstract}

Keywords: Electroencephalogram; Rat; Sleep; Wavelet transform

\section{INTRODUCTION}

Environmental heat is one of the well-known stressor to the mankind. Although, the problems of heat-afflicted illness are receiving increased importance in view of the current estimates of global warming and its impact on biological systems, the etiological factors that lead to heat exhaustion and heat stroke have not been well established. Review of literature revealed that the afflictions and damages to the central nervous system (CNS) and alterations in brain cortical potentials or electroencephalogram (EEG) [1] imposed by high environmental temperature have largely been ignored as the likely cause of heat induced mortality, although it is well known that neurochemical and cellular mechanisms of neural tissues are highly temperature sensitive [2].

Over the years, various signal processing techniques have been applied to the analysis of clinical EEG signals which are inherently dynamic, non-linear, stochastic and non-stationary [3-6]. Statistical pattern recognition was one of the first methods used for sleep-EEG analysis [7]. Following this method, quantitative features in terms of intervals were extracted from EEG and optimized [8]. A piece-wise segmentation and clustering techniques had also been developed, that was based on the assumption that an EEG consists of finite number of elementary patterns, which may be determined by dividing the signals a priori into segments of one second each [9]. Among several signal-processing techniques applied for EEG analysis, power spectrum analysis using the fast Fourier transform (FFT) was one of the most popular methods to estimate frequency and amplitude changes [10-13] in different pathological and psychological states. However, this approach considers the EEG signal as a stationary process, which assumption is not satisfied in practice, hence restricting the actual confidence on results. In contrary, the wavelet transform analysis enables to provide time-frequency information simultaneously, and effectively used in many biomedical signal analysis [14-17].

As time-frequency signal's analysis methods offer simultaneous interpretation of the signal in both time and frequency which allows local, transients or intermittent components to be elucidated $[18,19]$. Many of the ideas 
behind wavelet transforms have been in existence for a long time. A strong mathematical framework was built around the basic wavelet idea and is documented in the recent book by Mayer [20], which also shows the connections to earlier results in operator theory. Wavelet transform analysis has now been applied to a wide variety of biomedical signals including the electromyogram (EMG), EEG, clinical sounds, respiratory patterns and blood pressure trends [21].

Application of wavelet transform is found to be very much useful in automated analysis of medical signals and other signal processing tasks [22-27], automatic recognition of vigilance state, where prediction of the level of drowsiness was examined and delta, theta, alpha, and beta sub-frequencies of the EEG signals were extracted by using the wavelet transform technique [14]. In near past, variation of component powers in different frequency bands of rat electroencephalogram under slow wave sleep was also studied using wavelet transform [17]. Dynamic state recognition and event-prediction are fundamental tasks in biomedical signal processing. Therefore, motivated by adaptive time frequency patterns and data compression capabilities of wavelet transforms, a system has been made to identify the changes in sleep EEG spectral patterns due to exposure to high environmental heat.

\section{MATERIALS AND METHODS}

\subsection{Subjects and Electrode Implantation}

The experiments were carried out with male Charles Foster rats of age 12-14 weeks and weight around 180-200 grams at the beginning of the experiment. The rats were individually housed in polypropylene cages $(30 \mathrm{~cm} \times 20 \mathrm{~cm} \times 15 \mathrm{~cm}$ ) with drinking water and food (Hindustan Liver Limited, India) ad libitum. All rats were kept in an ambient environment temperature of 23 $\pm 1{ }^{\circ} \mathrm{C}$ from birth and the animal room was artificially illuminated with 12:12 hours Light: Dark cycle, changed at 07.00 hours and 19:00 hours Indian Standard Time (IST).

For the common grounding, midline Frontal stainless steel screw electrode, $1 \mathrm{~mm}$ in diameter, and two other similar screw electrodes were used for cortical EEG. Four stainless steel loop electrodes, insulated, except at the tip (two for electrooculogram (EOG) and two for EMG), were also used. Their socket contacts had earlier been prepared to a seven-pin amphitronic connector. Screw and loop electrodes were connected and soldered to the free pins of the connector, connected with thin flexible wires. The EEG and grounding screw electrodes were kept free; however, four pins of EOG and EMG electrodes were fixed in the amphitronics connector with the help of dental acrylic, well before implantation. Screw electrodes were connected and fixed to the socket contact by dental acrylic after fixing them on the skull.
Such separate connectors were used for each of the experimental animals for the recording of electrophysiological signals.

\subsection{Heat Stress Model}

The stress was produced in the rats, by subjecting them in the Biological Oxygen Demand (BOD) incubator (Oceania, India) at preset temperature of $38 \pm 1{ }^{\circ} \mathrm{C}$ and relative humidity $45-50 \%$ [33], simulated with the environmental conditions of Varanasi (India) in the months of May and June.

Acute heat stress: Rats were subjected to the incubator for continuous four hours of heat exposure from 8.00 a.m. to 12.00 p.m. for a single day, just before the recording of electrophysiological signals.

Chronic heat stress: Rats were subjected to the incubator for one hour daily for 21 days of chronic heat exposure from 8.00 a.m. to 9.00 a.m. and electrophysiological signals were recorded on $22^{\text {nd }}$ day.

Control: Respective control groups of rats were placed in the incubator at room temperature $\left(23 \pm 1^{\circ} \mathrm{C}\right)$ and whole procedure was followed exactly similar to that of their stressed groups.

\subsection{Electrophysiological Recordings}

The test chamber $(35 \mathrm{~cm} \times 25 \mathrm{~cm} \times 30 \mathrm{~cm})$ was constructed entirely of perspex and was located in a constantly illuminated (500-600 Lux white light), sound insulated chamber $(300 \mathrm{~cm} \times 180 \mathrm{~cm} \times 240 \mathrm{~cm})$. Holes at regular distances were made on the walls of test chamber for proper ventilation. The continuous four hours of recordings of EEG, EOG and EMG were performed from 12.00 hour to 16.00 hours IST on the recording day through the 8 channels Electroencephalograph (EEG-8, Recorders \& Medicare Systems, India). The paper recordings were performed with standard amplifier setup (Sinha, 2004) and at the chart speed of $7.5 \mathrm{~mm} / \mathrm{sec}$. The digitized data was collected, stored and processed with the help of data acquisition system (ADLiNK, 8112HG, NuDAQ, Taiwan) and processing software (Visual Lab.-M, Version 2.0c, Blue Pearl Laboratory, USA). The recordings were done with the sampling frequency of $256 \mathrm{~Hz}$ and selected data were stored in hard disk in small segments (approximately 2 minutes) in separate data files. Further, for ease of wavelet processing, recorded signals for all three states were split into an epoch of two seconds length.

\subsection{EEG Signal Processing with Wavelet}

Having acquired the digital data for sleep staging from different subjects, wavelet technique, which is based on multiresolution analysis (MRA), was applied on each signal. The data representing three sleep states such as AWAKE, rapid eye movement (REM) sleep and slow wave sleep (SWS) were selected from the raw EEG data 
recorded using VLM software and they were further subdivided into two seconds long epoch. Since the sampling frequency being $256 \mathrm{~Hz}$, one epoch comprises 512 data points. Using Matlab-7 (The Mathworks Inc.), all the epochs were loaded individually on Matlab's editor and converted to MAT files in Matlab's workspace.

Matlab codes calculate all the coefficients for each scale (1 to 128) and for each epoch containing 512 sample points, hence producing a matrix of size $[128,512]$. Daubechies order-4 wavelet was applied to AWAKE, REM, and SWS sleep EEG data of size [512,1] over scales 1:128, which gives coefficients as a function of time and scale. In order to know the frequency information contained in the signal instead of scales [16], following formula has been used: $F_{a}=\frac{F_{c}}{\Delta \cdot a}$.

where, $F_{a}$ is the pseudo frequency corresponding to scale ' $\mathrm{a}$ ' (in $\mathrm{Hz}$ ), $\mathrm{F}_{\mathrm{c}}$ is the center frequency or dominant frequency of a wavelet in $\mathrm{Hz}$, defined as the frequency with the highest amplitude in the Fourier transform of the wavelet function, and $\Delta$ is the sampling period. Given below are the frequencies corresponding to different levels of decomposition for $\mathrm{db}-4$ wavelet on scales over 1 to 128.

\begin{tabular}{|l|l|l|l|l|}
\hline Pseudo frequencies in $(\mathrm{Hz})$ & 3.7 & 7.9 & 16.6 & 30.4 \\
\hline Corresponding scales & 46 & 23 & 11 & 6 \\
\hline
\end{tabular}

Depending upon the desired frequency information, signals were grouped together into delta $(0.5-4 \mathrm{~Hz})$, theta (4-8 Hz), alpha $(8-14 \mathrm{~Hz})$, and beta $(14-30 \mathrm{~Hz})$ range. After extracting frequency information from the time domain signal and categorizing into delta, theta, alpha, and beta frequency bands, powers of all the four bands of AWAKE, REM and SWS were computed for each subject.

\subsection{Body Temperature}

Core body temperature was recorded as stress markers for both acute and chronic stress group of rats through the thermistor probe connected to 6-channel telethermometer. The marked probe at $4 \mathrm{~cm}$ was inserted to the rectum of the animal and kept static for 1 minute to record the body temperature. For acute stress group, body temperature was recorded before and after the heat exposure. While for the chronic stress group, the body temperature was recorded on every third day just before putting them into the incubator for chronic heat stress.

\section{RESULTS}

Variation of power in three vigilance states of sleep-EEG under chronic stressed condition has been observed as follows:

AWAKE: It is evident from Figure 1(a) that the wavelet coefficients posses considerably larger values between time instants 200-250 and about 475, for which scale vector spans $8-20$. The respective frequency band lies between $22.8 \mathrm{~Hz}$ to $9.13 \mathrm{~Hz}$. Here, the dominant frequency component that occurs at time instant 480 is 16.6 Hz, which is further followed by $12.2 \mathrm{~Hz}$ and other lower frequencies on their respective scales. Between 300 and 430, high frequency components disappear but delta and theta are easily detectable, where delta is found to be most dominant in this interval of time. The powers of delta, theta, alpha, and beta frequency bands of AWAKE state have been plotted as functions of time (samples) and frequency as shown in Figure 2(a), where subject belongs to its respective control group. Powers of all the frequency bands except beta were initially found to be almost equal (from higher to lower order of frequency in

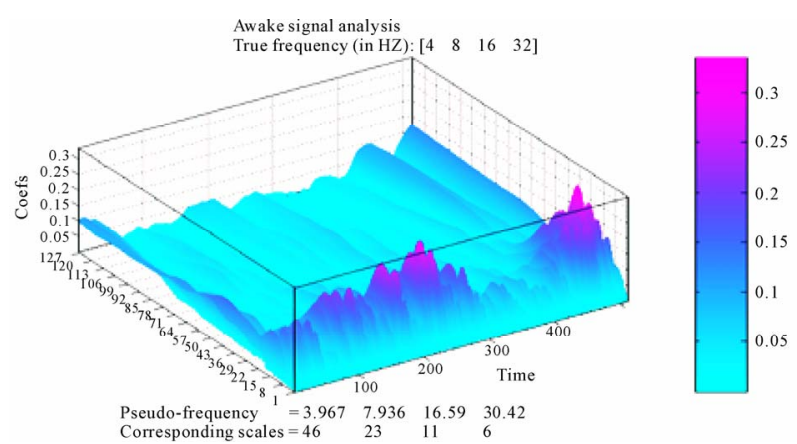

(a)

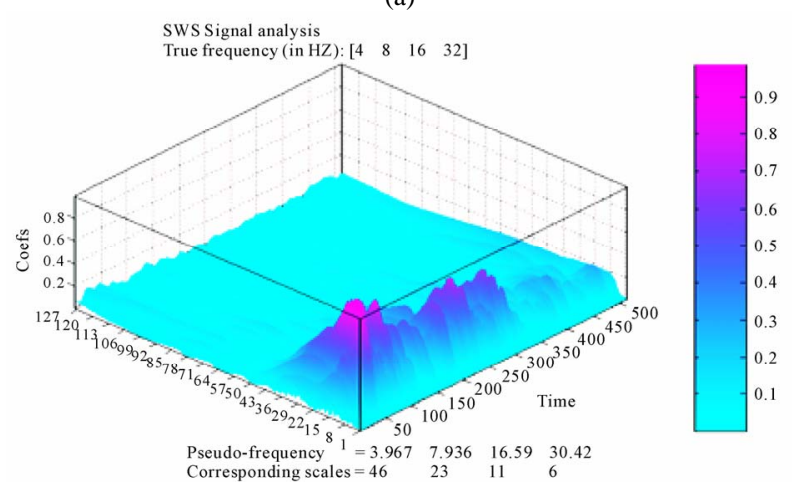

(b)

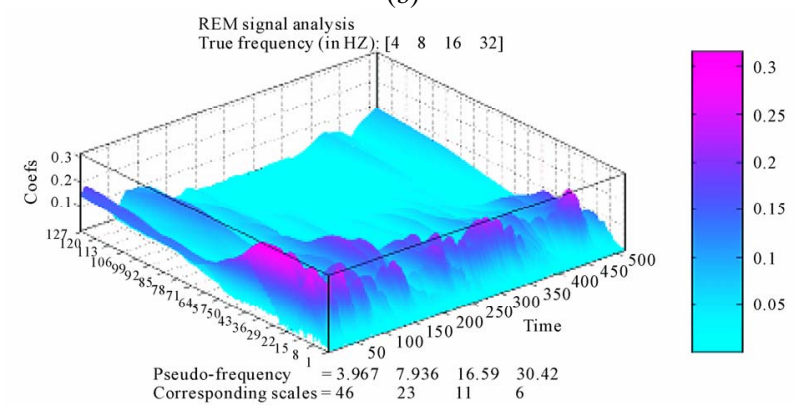

(c)

Figure 1. wavelet coefficients calculated and plotted over scales 1:512 (one epoch) for (a) AWAKE; (b) SWS; (c) REM signals subjected to chronic heat stress. 
the respective four bands), but for delta and theta bands, the lower frequency components posses more power than the components in the higher frequency side. It may also be seen that more than $40 \%$ of the durations, delta and theta bands have negligible power, however theta shows dominating spectrum than delta. It is quite obvious from the plot that power spectrum of AWAKE state shows both alpha and beta to be prominent bands for longer duration but higher frequencies of beta were found to have less power as compared to the lower one, whereas it reverses for alpha band. In the very beginning (between 0-50), theta seemed to have highest power. To study the variation in power, when subject is exposed to chronic heat stress, powers of different bands were plotted as shown in Figure 2(b). Some change in the powers of delta and beta components have been observed, however no change in powers of theta and beta was noted. Powers of the frequency components of delta band appeared to have decreased, whereas for beta band it has increased.

SWS: A plot of wavelet coefficients versus scale and time as shown in Figure 1(b) reveals time and frequency information regarding SWS signal taken from control group of chronic heat stress. From scales 1-20 and time interval 50-100, values of the wavelet coefficients are very high, which further showed decreasing trend. For delta, theta, and alpha bands, these values were very low after time instant 300. From Figure 3(a), it is clear that out of the three peaks lying in the time interval 50-100 and scales $15-22$, the middle one, which corresponds to frequency $10.2 \mathrm{~Hz}$ holds highest power, whereas the other two frequency components $(13 \mathrm{~Hz}$ and $8.3 \mathrm{~Hz})$ holds almost same power. The appearance of beta waves twice between time instants 150-200 and scales 7-10 has also been noted but found to have least power. During times 30-50 and 170-210, theta was seen in abundance. At many other places on higher scales, delta is present but its power is small. The plot investigating the changes in powers of four frequency bands of SWS signal recorded from subject under chronic heat stress has been shown in Figure 3(b). The effect of stress is not evident for theta and beta bands but variation in power can be seen for delta and alpha bands, which suggests that power of delta has increased in little and power of alpha being reduced. In this case, so far as power is concerned, delta and alpha happen to be the leading frequency bands. Any other remarkable change was not noticed for this epoch. The result reveals that for some periods, where delta has very small power, the other higher frequencies such as alpha and beta show significant amount of power. This analysis is also indicative of the fact that powers of frequencies in different frequency bands varied with time. In addition to this, powers of delta, theta, were very much less in more than quarter of EEG.
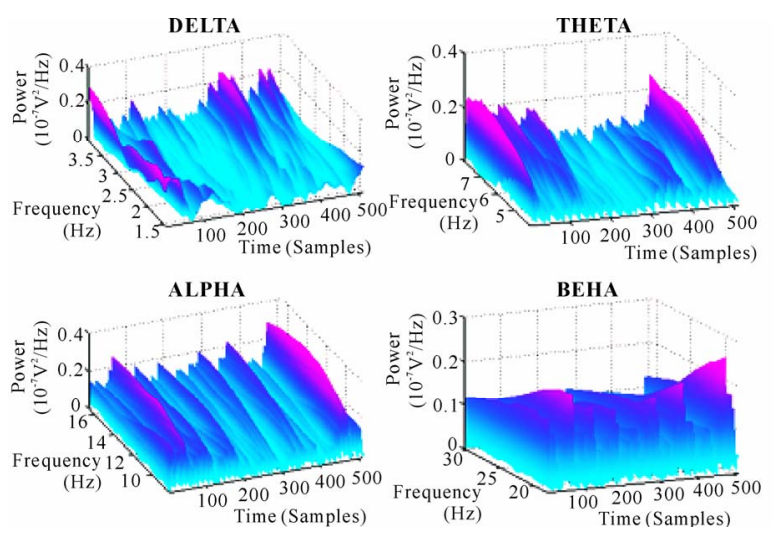

(a)
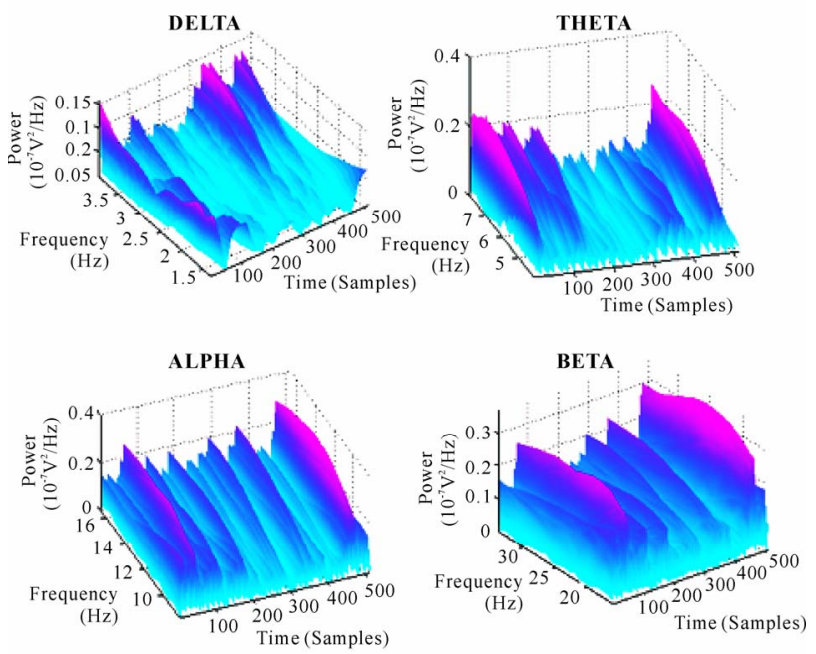

(b)

Figure 2. 3-D plots of power against time and frequency of delta, theta, alpha and beta bands of AWAKE signals under exposure to chronic heat stress. (a) control group, (b) respective chronic stressed group.

REM: Figure 1(c) depicts time and scale localization of frequency components of REM signals under chronic heat stress. Coefficient's values were found to be large between scales 7-67 and times 1-50 that covers all frequencies between $26-27 \mathrm{~Hz}$, while, within times 50-512, mostly faster waves were found to have larger values of the wavelet coefficients. At around 200, these coefficients for theta, alpha, and beta bands are quite insignificant but for delta, it is large. As regards the estimation of powers of the components of four bands in respective control group as shown in Figure 4(a), alpha is spread over almost the entire epoch with larger magnitude, all frequency components of beta band were initially found to be present with noticeable power but only the higher frequencies of delta band were seen in the beginning of the epoch with largest power. Components of delta showed poor presence except in the beginning of the epoch where, their powers for few components are comparable to alpha. When heat stress was given to the 
subject, the changes in powers of different bands have been shown in Figure 4(b). These changes can undoubtedly be seen for delta and beta bands. In case of stress, the overall power of delta band was found to be decreased, and for beta it has increased. No significant change in the powers of theta and alpha components were noticed. Powers of all the bands showed variations in time nonlinearly.

\subsection{Variation of Power Following Acute Heat Stress}

After the subjects were exposed to acute heat stress, the effect caused significant change in the powers of some frequency bands, whereas insignificant change in powers of other frequency bands was observed. Reports on the variation of powers of frequency components of delta, theta, alpha and beta bands in AWAKE, SWS and RAM states of sleep-EEG have been presented herein.
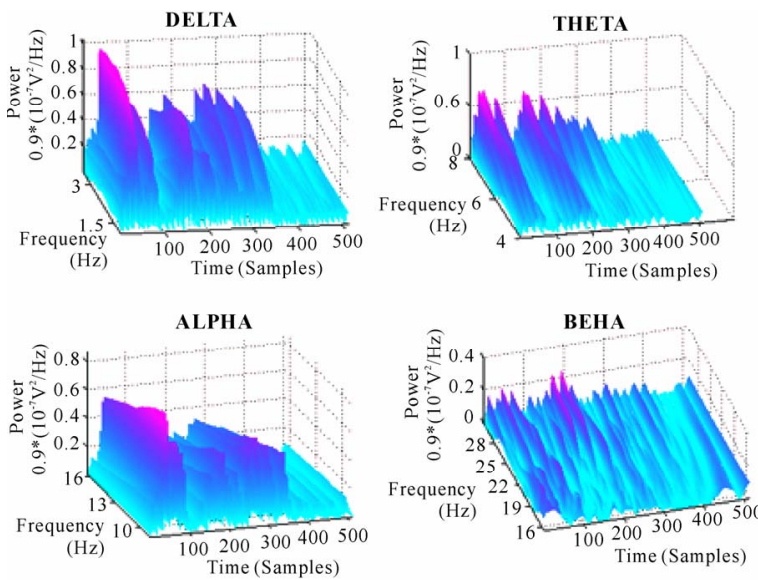

(a)
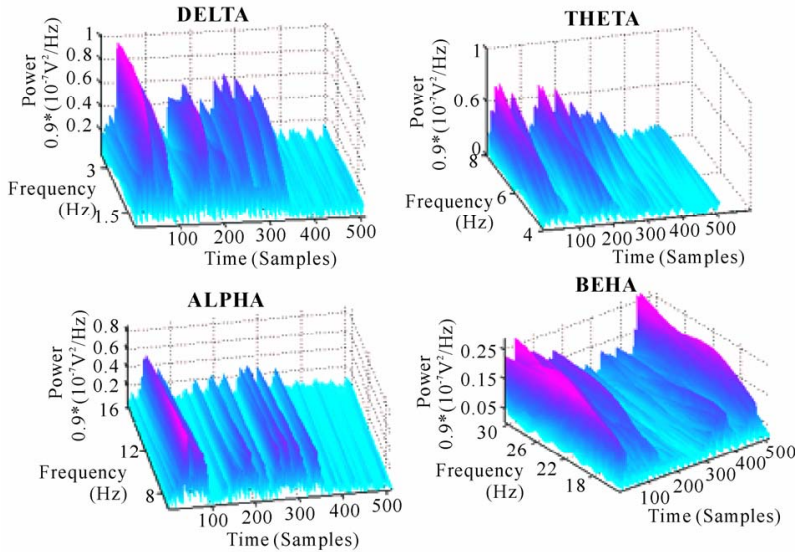

(b)

Figure 3. 3-D plots of power against time and frequency of delta, theta, alpha and beta bands of SWS signals under exposure to chronic heat stress. (a) control group; (b) respective chronic stress group.
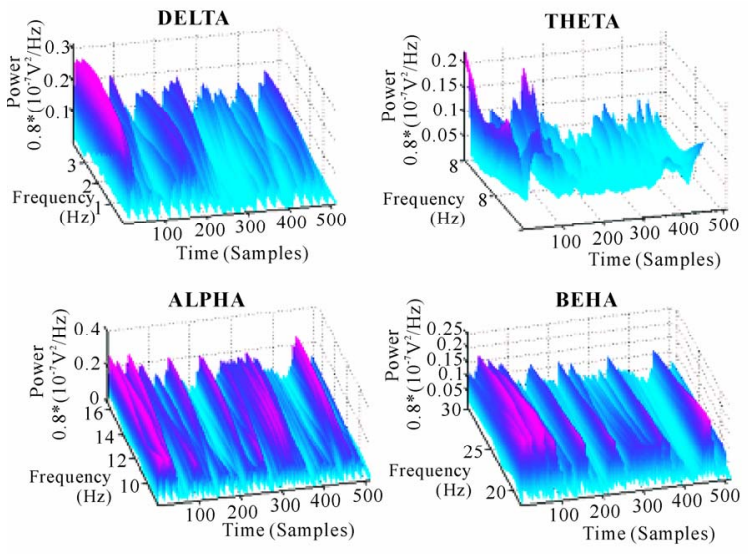

(a)
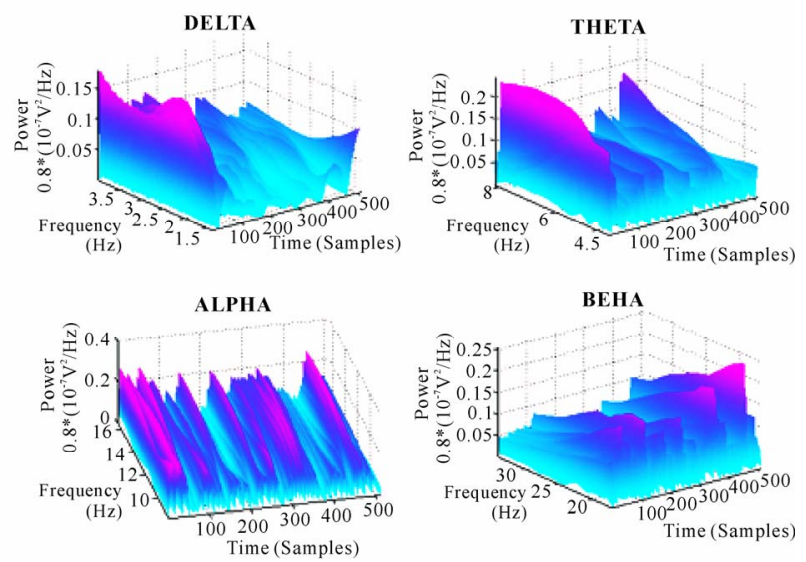

(b)

Figure 4. 3-D plots of power against time and frequency of delta, theta, alpha and beta bands of REM signals under exposure to chronic heat stress. (a) control group; (b) respective chronic stressed group.

AWAKE: Wavelet power spectrum as shown in Figure 5(a) suggest that higher frequency components of delta in the beginning of the epoch was seen to be higher and for the lower frequencies it gradually decreased. At the end of the epoch, change in this trend has been observed i.e., lower components of delta were high as compared to the upper one. In the middle of the epoch, delta's power was about half of the maximum power. Most of the time, theta components appeared to have greater power than delta and around the time instant 450, two major peaks were found whose powers were almost double the delta's power but on the other hand, in quarter of EEG, it remained very small for all frequencies of theta. All frequencies of alpha greater than $12 \mathrm{~Hz}$ acquired larger powers during time 100-250 and at the end too. Components of alpha and to some extent beta with considerable power were seen to exist for longer duration as compared to others. It was also witnessed that for the period, where power of delta was small, alpha band possessed large power. Frequent peaks of beta were no- 
ticed during times 100-300 in which lower components of beta were seen to hold more powers than the upper frequencies of the band. At time-480, powers of all the components were roughly equal and large (but less than alpha). So far as change in powers due to exposure of heat stress is concerned, delta, theta, and alpha bands showed negligible change, whereas the power of beta went up Figure 5(b).

SWS: As depicted in Figure 6(a), powers of higher frequencies of delta band among all the bands were found to be largest at time 50 i.e., at the start of the epoch, which further showed decreasing tendency over all the scales till the end of the epoch. Between times 300-512, it had drastically reduced but at the same time faster waves reported their existence with small powers. Next dominant frequency components belonged to theta band for which the highest component of the band held largest power. Theta too, did not show noticeable power during time 300-500. The plot of alpha implies that lower frequencies of alpha, which lies on scale 16-22 $(8.3 \mathrm{~Hz}-11.4 \mathrm{~Hz})$ seemed to have more power and it slightly decreases for the higher frequencies of the same

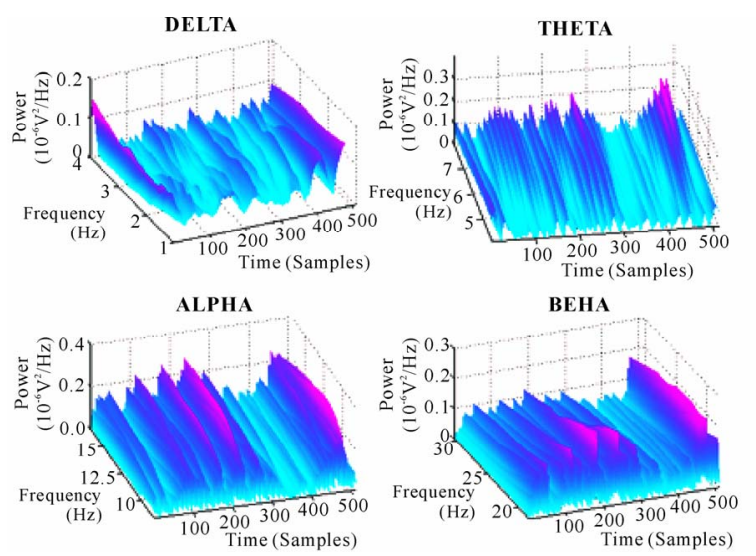

(a)
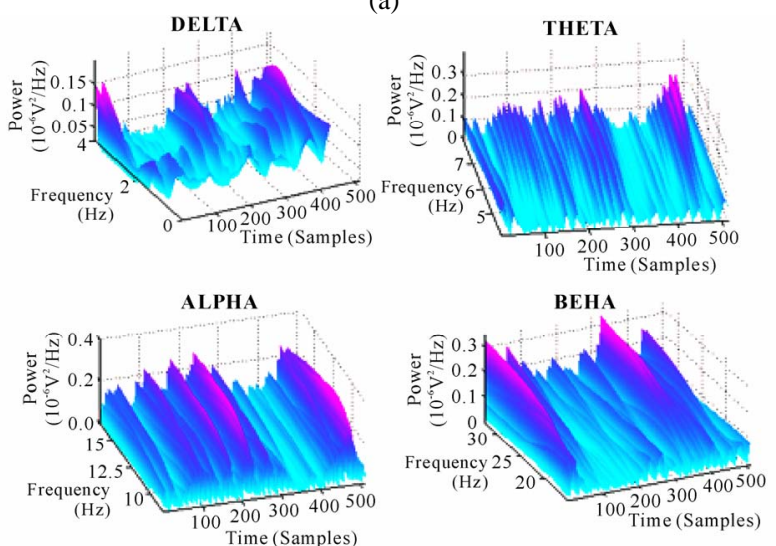

(b)

Figure 5. 3-D plots of power against time and frequenc of delta, theta, alpha and beta bands of AWAKE signals under exposure to acute heat stress. (a) control group; (b) respective acute stressed group. band. Between 220 and 340, many lower frequencies reported their presence with reduced power. After time 340 , powers of all frequency components were not worth mentioning. In beta band, two peaks were observed on scale-7 $(26.1 \mathrm{~Hz})$ during time 150-170 with equal power, which was quarter of delta power on scale-44. Figure 6(b) shows the power spectrum of respective stressed subjects. When subject undergoes exposure of acute heat stress, change in power over all scales was investigated for the three bands-delta, alpha, and beta. Increase in the powers of delta components were noticed at many places in the epoch but on the other hand, powers of alpha and beta components went down, indicating reciprocal relationship between changes in the powers of slow and fast waves.

REM: Having examined the power spectrum of all the four bands as shown in Figure 7(a), it may be noted that there existed more than $60 \%$ of time duration in which powers of faster waves (high frequencies of alpha band and low frequencies of beta band) were most significant. In the time interval 140-380 and scale 13-15 (12.2 Hz-14 Hz), powers of all the components were found to be
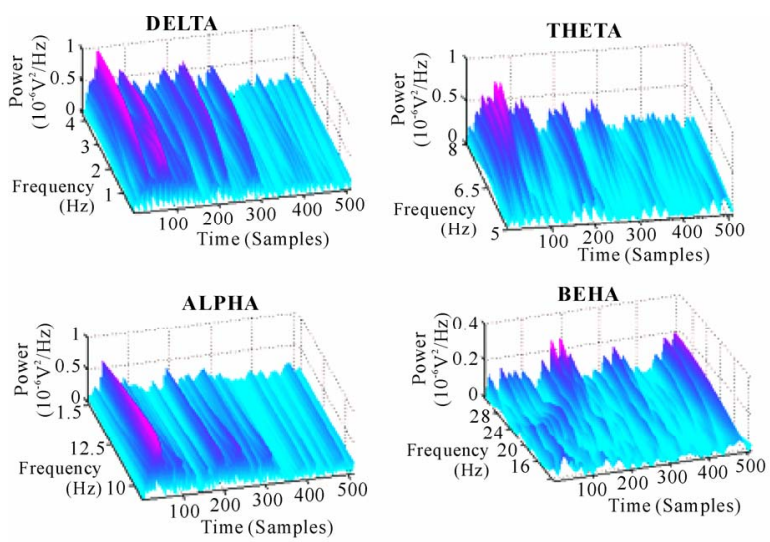

(a)
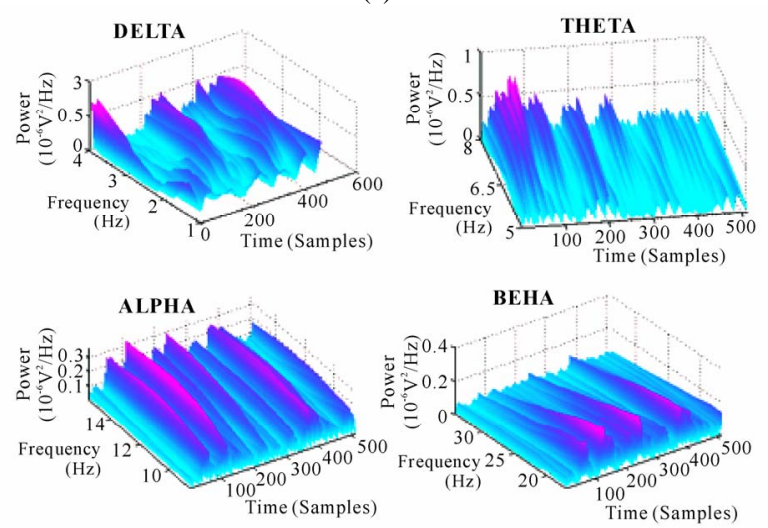

(b)

Figure 6. 3-D plots of power against time and frequency of delta, theta, alpha and beta bands of SWS signals under exposure to acute heat stress. (a) control group; (b) respective acute stressed group. 
large, which suddenly vanished for all the scales of the band after time instant 400. The same happened with beta too, but it did not agree with the power decay trend followed by the alpha components. Many peaks can clearly be seen between times 80-390 over all scales whose powers remained consistent. Like alpha band, sudden fall in powers of all the components were observed after time-390. Lot of inconsistency in variation of powers of delta components for all scales and time were marked. Up to time-200, increase in powers of frequency components from lower to higher order in delta band followed a gradual and unusual shift in time. In this band, powers of different components were found to be smaller than others. At time-220 and 300, two major peaks were obtained whose powers were seen to be at par with the average power of the alpha components. Over all scales, higher frequencies of theta contained more power than the lower one and decay in power was found to be almost uniform. The way acute heat stress alters the wavelet power spectra can unambiguously be seen in Figure 7(b). The alpha components witnessed a normal increase in the amount of power; meanwhile powers of beta components were significantly enhanced. Delta and theta components seemed to have nearly no change in their powers.

Similar computations by means of program written in Matlab were carried out for all the signals and analyses were performed for both the subjects stressed as well as their respective controls.

\subsection{Analysis of Changes in Body Temperature}

The results showed that acute heat exposure significantly increased the body temperature of rats. It was also observed that the increased body temperature of the animals returned to the control level after four to five hours of the incubation. The mean rectal temperatures recorded just before the incubation, for three weeks of chronically heat stressed rats were measured on every third day, just before daily exposure to $38 \pm 1^{\circ} \mathrm{C}$ for one hour. No change in body temperature was recorded during chronic stress till $3^{\text {rd }}$ day. The body temperature was found increased in young rats from $6^{\text {th }}$ day onwards. The analyses of results suggest significant increase in the mean rectal temperature of rats till $21^{\text {st }}$ day of chronic heat stress.

\section{Discussion}

The increase in body temperature is one of the main characteristics of the stress, induced by acute exposure of the high environmental heat. The body temperature of rats was significantly increased by acute heat stress similar to the findings of Menon et al [28], Sharma [29] and Sinha [34]. The review of literatures suggests that the immediate rise in the body temperature following acute heat stress plays essential role in the stimulation of
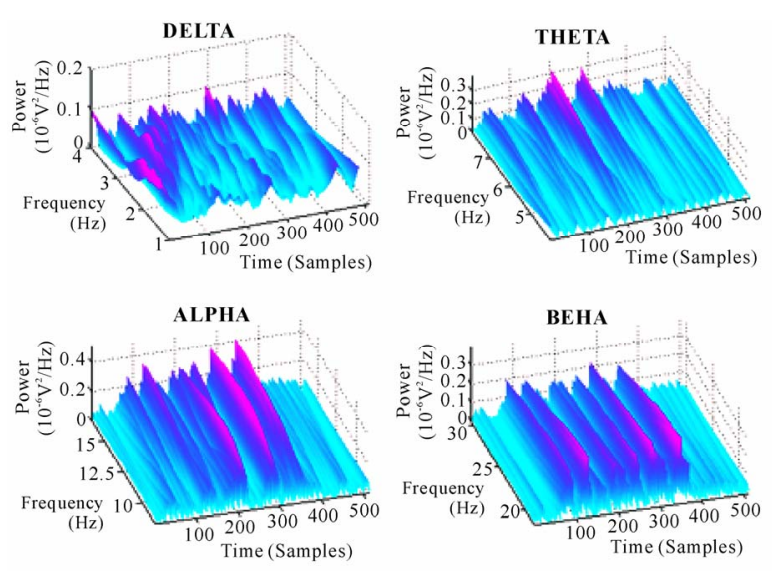

(a)
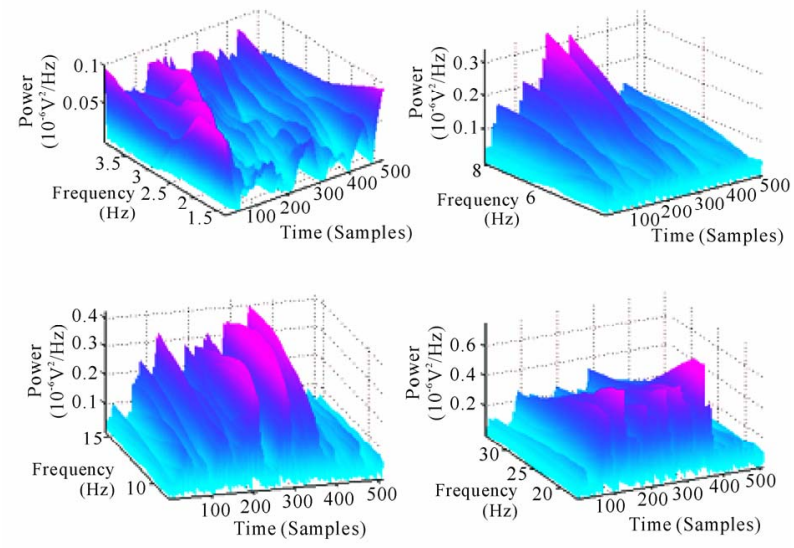

(b)

Figure 7. 3-D plots of power against time and frequency of delta, theta, alpha and beta bands of REM signals under exposure to acute heat stress. (a) control group; (b) respective acute stressed group.

the mechanisms necessary for heat dissipation. However, following the 21 days of chronic exposure of the high environmental heat, the body temperature of the rats was found to set at the higher temperature similar to the results obtained by Dey [30,31].

Wavelet analysis was performed to find out the dominant frequency components present in all bands- delta, theta, alpha, and beta for the subjects undergoing two types of heat stresses-acute and chronic and then the same was repeated for their respective control groups. Subsequent to the exposure of heat stress, all changes induced in frequency and power were investigated. Unlike other conventional techniques, the present study suggested that all the three sleep-stages-AWAKE, SWS and REM exhibited common characteristics regarding presence of leading frequency components in all four bands, which never remained constant at all times. Percentage power of different components of the four bands was always found to be varying at different intervals of time in the same signal of analysis. Thus, distribution of 
frequency and powers in these three vigilance states before and after the heat stress was applied, has been observed to be highly nonlinear and so comparative analysis is done every where instead of showing the results in exact figures. For all subjects when exposed to chronic heat stress, variation in the power of theta components were least observed, whereas changes in delta, alpha and beta were frequently observed for acutely exposed subjects. Effect of acute heat stress caused significant change in the powers of alpha and beta at many time intervals for REM state, but this change was noticed mostly for the lower frequency components of beta and higher components of alpha. The change induced was more noticeable (increased) for beta than that of alpha. For the same state, power of beta at some time instants was smaller than others. In AWAKE state, only beta components showed increase in power, however this change was smaller than what was seen for REM. Quantitatively, insignificant rise and fall of powers in other three bands-delta, theta, and alpha were observed. With regard to percentage power of theta, it seemed to hold more compared to delta. The analysis reflected that chronic heat stress caused power of frequency component of delta to decrease in AWAKE and REM states, whereas the least change in theta and alpha was noticed.

No report has come to light on the study of brain cortical electrical activity with the similar model of acute or chronic heat stress using wavelet analysis. However, it is evident that the changes found in the EEG activities following acute heat stress are similar to the earlier report [32]. It has been supposed that acute heat stress alters the EEG frequencies that may have occurred due to neuronal and non-neuronal changes in the CNS [32,33]. In the present work, the quantitative changes in EEG for four defined EEG frequency bands were done and it was found that even though the EEG power spectrum showed the recovery in four hours of EEG recording following acute heat exposure, the quantitative analysis of EEG still showed significant changes in EEG signals in all the three sleep-wake states. Conversely, the chronic heat stress showed similar irreversible changes in EEG power spectra as reported in the previous work. The quantitative EEG changes following chronic heat exposure has also been found to be strikingly similar to those reported by Sarbadhikari et al. [12] in their study on exercise stress. They showed that the chronic exercise stress increases beta activities and decreases delta activity in AWAKE state. In SWS, the beta activities were also found to have increased. Like the chronic exercise stress, long-term exposure to high environmental heat also increased the beta activities in all subjects and decreased the delta activity in AWAKE condition. The increased beta activities in SWS were also found to exist. These changes in EEG activities following chronic heat exposure seemed to be due to adaptations of animal's physiological systems to the new ambient environmental conditions [34].

Dubois et al. [32] reported an initial increase in EEG frequency with increase in body temperature either by spontaneous or artificially induced fever. If the elevation of body temperature was maintained long enough or above $41-42^{\circ} \mathrm{C}$, a major transient reduction in EEG activity was observed. They also showed that as cooling was resumed; these changes in EEG frequencies were usually totally reversible. Rarely, the changed EEG frequencies did not return to the control level and that may occur due to CNS damage, which attributed to anorexia, dehydration, metabolic imbalance, energy failure or cellular changes following heat stress [32,33]. Similar to this report, our findings showed changes in the beta frequency components after acute heat stress that returned to the control level in four hours of cooling at room temperature except in AWAKE state. However, beta and alpha frequency components in SWS did not return to the control level, which might reflect the neuronal and non-neuronal changes in the brain due to acute heat stress [32,33]. On the other hand, 21 days of chronic heat exposure significantly decreases the beta frequencies in AWAKE state in all four hours of EEG recording. The EEG recordings also indicate that the beta frequency components were decreased in second hour of SWS and third hour in REM sleep. It has been supposed that the changed EEG activity were recorded due to adaptations of animals physiological systems to the new ambient environmental conditions similarly as suggested by Sarbadhikari and his co-workers [12] in their study on chronic exercise stress. In the present study, it has been found that the changes in EEG components in three sleep-wake states in generalization are observed to be sensitive to hot environment and found dependent upon the different sleep-wake states, both acute and chronic heat stress conditions in all three sleep-wake cycle in all experimental groups of rats. Further, the results demonstrate that the wavelet analysis of long term EEG recordings can be used for obtaining useful results in analyzing heat induced changes in electrophysiological activities of cerebral cortex.

Several research works have been reported in the area of EEG signal analysis using wavelet transform as a preprocessor such as sleep spindles detection, spike detection, sleep EEG analysis, event related potentials, epileptic seizures, but no work has been reported so far which investigated the changes in frequency and powers of EEG due to heat stress, with the help of wavelet transform. However, with this existing model no work has been reported except by Sinha and Ray [34], and Sinha [1], who investigated these changes by applying Fourier transform to the time domain EEG signals. Since Fourier transform has got some limitations due to which the analysis contains only globally averaged information. Transients EEG phenomena, which occur for short dura- 
tion were not detected. In the earlier works, it has only been shown what the dominant frequency components in these sleep-states are, and how they change after heat stress. It was also shown that four frequency subbands for any particular epoch show constant behavior. In the present study, it has been clearly demonstrated the presence of different components with their varying powers in their separate bands with time of their occurrence. Information regarding frequency and time are very much localized. This study in the time-frequency domain separates the signal's power in different frequency bands with respect to time and frequency. Hence, wavelet technique provides superior resolution on data analyses at a lower time scale and thus can provide more refined data analyses on sleep EEG data compared to traditional Fourier analysis. This paper provides an example applying this technique to physiological data. Hope this technique can have broader application in sleep state transition analyses.

\section{REFERENCES}

[1] Sinha, R.K. (2004) Electro-encephalogram disturbances in different sleep-wake states following exposure to high environmental heat. Medical \& Biological Engineering \& Computing, 42, 282-287.

[2] Sinha, R.K. (2007) Study of changes in some pathophysiological stress markers in different age groups of an animal model of acute and chronic heat stress. Iranian Biomedical Journal, 11, 101-111.

[3] Guler, I., Kiymik, M.K., Akin, M. and Alkan, A. (2001) AR spectral analysis of EEG signals by using maximum likelihood estimation. Computers in Biology and Medicine, 31, 441-450.

[4] Herrmann, C.S., Arnold, T., Visbeck, A., Hundemer, H.P. and Hopf, H.C. (2001) Adaptive frequency decomposition of EEG with subsequent expert system analysis. Computers in Biology and Medicine, 31, 407-427.

[5] Peters, B.O., Pfurtscheller, G. and Flyvbjerg, H. (2001) Automatic differentiation of multichannel EEG analysis. IEEE transactions on Biomedical Engineering, 48, 111-116.

[6] Vuckovick, A., Radivojevic, V., Chen A.C.N. and Popovic, D. (2002) Automatic recognition of alertness and drowsiness from EEG by an artificial neural network. Medical Engineering and Physics, 24, 349-360.

[7] McKeon, M.J., Humphries, C., Achermann, P., Borbely, A.A. and Sejnowski, T.J. (1997) Anew method for detecting state changes in EEG: Exploratory application to sleep data. Journal of Sleep Research, 7, 48-56.

[8] Dement, W.C. and Kleitman, N. (1957) Cyclic variations in EEG during sleep and their relation to eye movements, body motility and dreaming. Electroencephalography and Clinical Neurophysiology, 9, 673-690.

[9] Jansen, B.H., Hasman, A. and Lenten, R. (1981) Piecewise EEG analysis: An objective evaluation, International Journal of Bio-Medical Computing, 12, 17-27.

[10] Al-Nashash, H.A.M. (1995) A dynamic Fourier series for the compression of ECG using FFT and adaptive coefficient estimate. Medical Engineering and Physics, 17,
197-203.

[11] Sinha, R.K. (2003) Artificial neural network detects changes in electro-encephalogram power spectrum of different sleep-wake states in an animal model of heat stress. Medical \& Biological Engineering \& Computing, 41, 595-600.

[12] Sarbadhikari, S.N., Dey, S., Ray, A.K. (1996) Chronic exercise alters EEG power spectra in an animal model of depression. Indian Journal of Physiology and Pharmacology, 40, 47-57.

[13] Jung, T.P., Makeig, S., Stensmo, M. and Sejnowski, T.J. (1997) Estimating alertness from the EEG power spectrum. IEEE Transactions on Biomedical Engineering, 44, 60-69.

[14] Subasi, A. (2005) Automatic recognition of alertness level from EEG by using neural network and wavelet coefficients. Expert Systems with Applications, 28, 701-711.

[15] Adeli, H. et al. (2007) A wavelet-chaos methodology for analysis of EEGs and EEG Subbands to detect seizure and epilepsy. IEEE Transactions on Biomedical Engineering, 54(2), 205-211.

[16] Adeli, H., Zhou, Z. and Dadmehr, N. (2003) Analysis of EEG records in an epileptic patient using wavelet transform. Journal of Neuroscience Methods, 123, 69-87.

[17] Feng, Z. and Xu, Z. (2002) Analysis of rat electroencephalogram under slow wave sleep using wavelet transform. Proceedings of the Second Joint EMBS/BMES Conference Houston, TX, USA.

[18] Daubechies, I. (1990) The Wavelet transform time-frequency localization and signal analysis. IEEE Transactions on Information Technology, 36(5), 961-1005.

[19] Mallat, S. (1989) A theory for multiresolution signal decomposition: The wavelet representation. IEEE Transactions on Pattern Analysis and Machine Intelligence, 11(7), 674-693.

[20] Meyer, Y. (1989) Orthonormal wavelets in Wavelets, Time-Frequency Methods and Phase Space (Lecture Notes on IPTI), J. M. Combes et al., Ed., New Work: Springer-Veriag.

[21] Bianchi, A.M., Mainardi, L.T. and Cerutti, S. (2000) Time-frequency analysis of biomedical signals. Transactions of the Institute of Measurement and Control, 22, 321-336.

[22] Kalayci, T. and Ozdamar, O. (1995) Wavelet preprocessing for automated neural network detection of EEG spikes. IEEE Engineering in Medicine and Biology Magazine, 14, 160-166.

[23] Farge, M. (1992) Wavelet transforms and their applications to turbulence. Annual Review of Fluid Mechanics, 24, 395-457.

[24] Sakkalis, V. et al. (2006) Significant EEG features involved in mathematical reasoning: Evidence from wavelet analysis. Brain Topography, 19(1/2), 53-60.

[25] Shen, M., Sun, L. and Chan, F.H.Y. (2001) Method of extracting time-varying rhythms of electroencephalography via wavelet packet analysis. IEE Proceedings-Science, Measurement and Technology, 148.

[26] Ting, W. et al. (2007) EEG feature extraction based on wavelet packet decomposition for brain computer interface. Meaurement.

[27] Unser, M. and Aldroubi, A. (1996) A review of wavelets in biomedical applications. Proceedings of the IEEE, 
84(4), 626-638.

[28] Menon, M.K. and Dandiya, P.C. (1969) Behavioural and brain neurohormonal changes produced by acute heat stress in rats: Influence of psychopharmacological agents. European Journal of Pharmacology, 8, 284-291.

[29] Sharma, H.S. (1982) Blood-brain barrier (BBB) in stress. $\mathrm{Ph}$. D. Thesis, Zoology, Banaras Hindu University.

[30] Dey, P.K. (1998) Modification of dopamine receptor agonist mediated behavioral responses in rats following exposure to chronic heat stress. Biomedicine, 18, 41-47.

[31] Dey, P.K. (2000) Involvement of endogenous opiates in heat stress. Biomedicine, 20, 143-148.

[32] Dubois, M., Sato, S., Lees, D.E., Bull, J.M., Smith, R.,
White, B.G., Moore, H. and Macnamara, T.E. (1980) Electroencephalographic changes during whole body hyperthermia in humans. Electroencephalography and Clinical Neurophysiology, 50, 486-495.

[33] Sharma, H.S., Westman, J. and Nyberg, F. (1998) Pathophysiology of brain edema and cell changes following hyperthermic brain injury: Progress in Brain Research. Elsevier, Amsterdam, 115, 351-412.

[34] Sinha, R.K. and Ray, A.K. (2004) An assessment of changes in open-field and elevated plus-maze behavior following heat stress in rats. Iranian Biomedical Journal, 8, 127-133. 